\title{
Desempenho da saúde pública no semiárido brasileiro
}

A saúde desempenha um papel essencial para o desenvolvimento sustentável. Populações que vivem em ambientes saudáveis são mais sadias e produtivas e têm maiores condições de promover e sustentar o desenvolvimento econômico, social e ambiental. O objetivo geral deste estudo é analisar o desempenho da saúde pública no semiárido brasileiro. Os objetivos específicos são: caracterizar a região com relação a indicadores de recursos humanos e financeiros, de atenção integral à saúde da mulher e da criança, de redução dos riscos e agravos à saúde da população, de acesso potencial da atenção básica, de acesso obtido na atenção ambulatorial e hospitalar de média e alta complexidade, de efetividade da atenção básica e de despesas com saúde e calcular o Índice de Desenvolvimento da Saúde (IDS). Foram utilizados dados obtidos através do Sistema de Informação de Vigilância da Qualidade da Água para Consumo Humano (SISAGUA), Departamento de Informática do SUS (DATASUS) e Sistema de Informações sobre Orçamentos Públicos em Saúde (SIOPS) para o ano de 2011. O tratamento dos dados foi realizado com as técnicas de análise fatorial e análise de conglomerados. Com base nos resultados do IDS, pode-se concluir que a maioria dos municípios do semiárido apresenta baixo desenvolvimento da saúde. Uma minoria ínfima dos municípios da região está enquadrada nas faixas de médio e alto desenvolvimento da saúde.

Palavras-chave: Saúde Pública; Desenvolvimento; Semiárido.

\section{Performance of public health in the Brazilian semiarid}

\begin{abstract}
Health plays a key role for sustainable development. Populations living in healthy environments are healthier and more productive and are better able to promote and sustain economic, social and environmental development. The general objective of this study is to analyze the public health performance in the Brazilian semiarid region. The specific objectives are: to characterize the region in relation to indicators of human and financial resources, comprehensive health care for women and children, risk reduction and health problems for the population, potential access to basic health care, access in medium and high complexity outpatient and hospital care, in the effectiveness of basic care and in health expenditure, and to calculate the Health Development Index (IDS). Data were obtained through the Information System for Monitoring Water Quality for Human Consumption (SISAGUA), Department of Information Technology of SUS (DATASUS) and Information System on Public Budgets in Health (SIOPS) for the year 2011. The treatment of the data was carried out with the techniques of factorial analysis and analysis of conglomerates. Based on the results of IDS, it can be concluded that the majority of municipalities in the semiarid region show low health development. A tiny minority of the municipalities of the region is framed in the middle and high health development bands.
\end{abstract}

Keywords: Public Health; Development; Semiarid

Topic: Desenvolvimento, Sustentabilidade e Meio Ambiente

Reviewed anonymously in the process of blind peer.
Received: $10 / 06 / 2018$

Approved: 24/07/2018

Nagilane Parente Damasceno (D)

Universidade Regional do Cariri, Brasil

http://lattes.cnpq.br/5377710567483058

http://orcid.org/0000-0002-2042-0024

nagilaneparente@yahoo.com.br

\section{Ahmad Saeed Khan}

Universidade Federal do Ceará, Brasil

http://lattes.cnpq.br/3198350508846033

saeed@ufc.br

Patrícia Verônica Pinheiro Sales Lima

Universidade Federal do Ceará, Brasil

http://lattes.cnpq.br/7172491133426747

pvpslima@gmail.com

\section{Referencing this:}

DAMASCENO, N. P.; KHAN, A. S.; LIMA, P. V. P. S.. Desempenho da saúde pública no semiárido brasileiro. Revista Ibero Americana de Ciências Ambientais, v.9, n.5, p.171-187, 2018. DOI:

http://doi.org/10.6008/CBPC2179-6858.2018.005.0016 


\section{INTRODUÇÃO}

A saúde é considerada uma das áreas mais importantes para o crescimento e desenvolvimento econômico, tanto como condição para o pleno exercício dos direitos humanos quanto como demandante de uma complexa cadeia de bens e serviços de tecnologia avançada. $O$ direito à saúde, como parte do dever do estado, acrescenta um papel essencial às políticas públicas, buscando expandir o acesso da população a esses produtos (PIMENTEL et al., 2012).

De acordo com o Relatório da Comissão sobre Macroeconomia e Saúde, fatores como esperança de vida mais longa e mortalidade infantil baixa estão associados ao desenvolvimento econômico. Indivíduos saudáveis são mais produtivos; os bebês e as crianças saudáveis podem se desenvolver melhor e se tornar adultos bem-sucedidos. Ou seja, uma população saudável pode contribuir positivamente para o desenvolvimento econômico do país. Segundo o Relatório, a ampliação dos investimentos em saúde pode gerar uma renda suplementar a ser utilizada para melhorar as condições de vida e a infraestrutura social nos países mais pobres (SACHS, 2001).

Os países com desenvolvimento humano muito elevado apresentam uma evolução positiva da renda e indicadores com resultados extremamente favoráveis nas áreas de saúde e educação. No âmbito da saúde, esses países têm, em geral, altas taxas de cobertura vacinal e baixas taxas de mortalidade infantil. Além disso, mortes em decorrência de doenças como a cólera e a malária não costumam ocorrer (PNUD, 2013a).

A relação entre saúde e desenvolvimento foi evidenciada no contexto dos Objetivos de Desenvolvimento do Milênio (ODM), uma vez que três deles são diretamente ligados à saúde: reduzir a mortalidade infantil, melhorar a saúde materna e combater o HIV/Aids, a malária e outras doenças (PNUD, 2006). O reconhecimento da associação entre saúde e desenvolvimento sustentável conduziu à incorporação de metas relacionadas à saúde dentro dos Objetivos de Desenvolvimento Sustentável (ODS).

O terceiro ODS consiste em assegurar uma vida saudável e promover o bem-estar para todos em todas as fases da vida e é composto por nove metas. Conforme salientam Buss et al. (2016), vários ODS contêm metas associadas à saúde, o que mostra a incorporação, por parte do Grupo de Trabalho Aberto sobre Objetivos de Desenvolvimento Sustentável, da estratégia de incluir a saúde em todas as políticas.

A relação existente entre ausência de saúde e persistência da pobreza é ainda mais direta. As populações pobres são mais propensas a contrair doenças devido à dificuldade de acesso à água potável, saneamento básico, informações sobre ações de prevenção e controle de doenças e alimentação apropriada. Por outro lado, as enfermidades tendem a aprofundar a pobreza, tanto pelo custo financeiro associado ao tratamento da doença, quanto por reduzir a capacidade laboral do indivíduo (PIMENTEL et al., 2012).

A promoção e a prevenção da saúde são fundamentais para o bem-estar do ser humano e para o desenvolvimento socioeconômico sustentável. O acesso aos cuidados de saúde no momento adequado se configura como uma das principais formas de promover e manter a saúde. Entretanto, esse acesso é crítico e não pode ser garantido sem a existência de um sistema de financiamento de saúde eficiente, que possibilite 
aos indivíduos utilizar os serviços de saúde quando necessário, sem a realização de sacrifícios financeiros expressivos, assegurando a cobertura universal (WHO, 2010).

O semiárido brasileiro, área de estudo desta pesquisa, apresenta os índices de pobreza mais elevados do país e desempenho insatisfatório nos seus indicadores sociais. De acordo com informações do Atlas do Desenvolvimento Humano no Brasil, 59,48\% dos municípios da região apresentaram baixo IDH em 2010 e nenhum deles atingiu a faixa mais elevada do índice (SÁ et al., 2012; PNUD, 2013b).

O objetivo geral deste estudo é analisar o desempenho da saúde pública no semiárido brasileiro. Os objetivos específicos são: caracterizar a região com relação a indicadores de saúde selecionados e calcular o Índice de Desenvolvimento da Saúde (IDS), de modo a possibilitar o agrupamento e a hierarquização dos municípios com relação ao desempenho na saúde pública.

Apesar da importância da relação existente entre a saúde e as dimensões econômica, social e ambiental do desenvolvimento sustentável, pouco se conhece sobre a situação da saúde no semiárido brasileiro em virtude da escassez de estudos relacionados ao tema. Desse modo, é imprescindível investigar profundamente a realidade das condições de saúde da região. Este estudo examina o desempenho da saúde pública através do IDS, importante instrumento para avaliar ações políticas e orientar gestores no intuito de que um sistema de saúde mais adequado, que atenda às necessidades prioritárias das populações dos municípios que compõem a área de estudo, possa ser disponibilizado.

\section{METODOLOGIA}

\section{Análise Fatorial}

A análise fatorial é uma técnica multivariada de interdependência empregada para viabilizar o estudo de eventuais inter-relações entre um grande número de variáveis, com o objetivo de condensar a informação contida em certo número de variáveis originais em um conjunto menor de variáveis estatísticas (fatores), com uma perda mínima de informação (HAIR et al., 2005). De acordo com Johnson et al. (2007), na análise fatorial, as variáveis são agrupadas em função de suas correlações. Conforme Fávero et al. (2009), o modelo de análise fatorial pode ser apresentado da seguinte forma:

$$
X_{i}=a_{i 1} F_{1}+a_{i 2} F_{2}+\ldots+a_{i m} F_{m}+\varepsilon_{i} \quad(i=1, \ldots, p)
$$

Em que: $X_{i}=$ variáveis padronizadas; $a_{i}=$ cargas fatoriais, que medem a importância dos fatores na composição de cada variável (correlação); $F_{m}=$ fatores comuns; $\varepsilon_{i}=$ erros ou fatores específicos.

De acordo com Fávero et al. (2009), os fatores podem ser estimados através da combinação linear das variáveis, do seguinte modo:

$$
\begin{aligned}
& F_{1}=d_{11} X_{1}+d_{12} X_{2}+\ldots+d_{1 m} X_{i} \\
& F_{2}=d_{21} X_{1}+d_{22} X_{2}+\ldots+d_{2 m} X_{i} \\
& \vdots \\
& F_{m}=d_{m 1} X_{1}+d_{m 2} X_{2}+\ldots+d_{m i} X_{i}
\end{aligned}
$$

Em que: $F_{m}=$ fatores comuns; $d_{m i}=$ coeficientes dos escores fatoriais e $X_{i}=$ variáveis originais. A partir da carga fatorial pode-se obter a comunalidade. A comunalidade representa a proporção da variância de $X_{i}$ explicada pelo fator comum e é dada pelo quadrado da carga fatorial. O escore fatorial, por 
sua vez, é obtido por meio da multiplicação dos coeficientes $d_{m i}$ pelo valor das variáveis originais. Caso exista mais de um fator, o escore fatorial corresponderá às coordenadas da variável em relação aos eixos ou fatores (HAIR et al., 2005).

Para verificar a adequabilidade da técnica de análise fatorial, pode ser utilizado o teste de esfericidade de Bartlett e a estatística de Kaiser-Meyer-Olkin (KMO). O teste de esfericidade de Bartlett é utilizado para testar se a matriz de correlações é uma matriz s identidade com determinante igual a 1. Se a matriz de correlações for igual à matriz identidade, as variáveis não estão correlacionadas e, portanto, a análise fatorial não deve ser utilizada (FÁVERO et al., 2009).

A estatística $K M O$, cujos valores variam entre 0 e 1, é empregada para verificar a adequação da amostra com relação ao grau de correlação parcial entre as variáveis. Um valor de $K M O$ próximo a 0 mostra que a análise fatorial não é adequada, pois existe uma correlação fraca entre as variáveis. Por outro lado, quanto mais próximo de 1 for o seu valor, mais adequado é o emprego da técnica. Valores para a estatística KMO superiores a 0,6 indicam que a análise fatorial é adequada (FÁVERO et al., 2009). A estatística KMO, segundo Hair et al. (2005), é calculada através da seguinte expressão:

$$
K M O=\frac{\sum_{i \neq j} \sum r_{i j}^{2}}{\sum_{i \neq j} \sum r_{i j}^{2}+\sum_{i \neq j} \sum a_{i j}^{2}}
$$

Em que: $r_{i j}=$ coeficiente de correlação entre variáveis; $a_{i j}=$ coeficiente de correlação parcial.

Também foi analisada a Medida de Adequação da Amostra ou Measure of Sampling Adequacy (MSA) para cada variável. De acordo com Fávero et al. (2009), valores acima de 0,50 para uma variável individual indicam adequação da aplicação da análise fatorial. A MSA é calculada da seguinte forma:

$$
M S A=\frac{\sum_{i \neq j} r_{i j}^{2}}{\sum_{i \neq j} r_{i j}^{2}+\sum_{i \neq j} a_{i j}^{2}}
$$

Os fatores foram extraídos através da Análise dos Componentes Principais (ACP) que, segundo Hair et al. (2005), possibilita a redução dos dados para obtenção do mínimo número de fatores necessários para explicar o máximo de variância representada pelas variáveis originais. O critério da raiz latente (critério de Kaiser) é utilizado para a escolha do número de fatores. De acordo com esse critério, escolhe-se o número de fatores a reter com base no número de valores próprios (eigenvalues) acima de 1. Os eigenvalues apresentam a variância explicada por cada fator, isto é, quanto cada fator consegue explicar da variância total (FÁVERO et al., 2009).

Para a rotação dos fatores foi utilizado o método Varimax, que procura minimizar o número de variáveis que possuem altas cargas em um fator, o que simplifica a interpretação dos fatores. Finalmente, os fatores foram interpretados e nomeados através das cargas fatoriais. As cargas fatoriais maiores que 0,30 atingem o nível mínimo; cargas de 0,40 são consideradas mais importantes; cargas superiores a 0,50 são 
consideradas estatisticamente significativas. As variáveis com cargas mais elevadas influenciam mais a seleção de um nome ou rótulo para representar um fator (HAIR et al., 2005).

\section{Índice de Desenvolvimento da Saúde (IDS)}

A partir dos fatores extraídos e das suas respectivas cargas fatoriais, obtidos através da aplicação da análise fatorial, foi possível estudar o nível de desenvolvimento da saúde nos municípios do semiárido brasileiro. Desse modo, os fatores extraídos, representativos do desempenho da saúde pública, foram utilizados para calcular o IDS, que representa o nível de desenvolvimento da saúde na região. O cálculo do IDS foi realizado com base na metodologia proposta por Lemos (2000). Assim, os fatores extraídos foram agregados através da seguinte equação:

$$
I D S_{i}=\sum_{j=1}^{n} \frac{\lambda_{j}}{\sum \lambda_{j}} F_{j i}^{*}
$$

Em que: IDS é o índice do i-ésimo município; $j$ é a j-ésima raiz característica; $n$ é o número de fatores extraídos na análise; $F_{j i}^{*}$ é o j-ésimo escore fatorial do i-ésimo município; $\sum \lambda_{j}$ é o somatório das raízes características referentes aos $\mathrm{n}$ fatores extraídos.

De modo a tornar todos os valores dos escores fatoriais (Fji) superiores ou iguais a zero, todos eles foram colocados no primeiro quadrante antes do cálculo do IDS, utilizando-se a seguinte expressão algébrica, sugerida por Lemos (2000):

$$
F_{j i}^{*}=\frac{F_{j i} F_{j}^{\min }}{F_{j}^{\max } F_{j}^{\min }}
$$

Em que: $F_{j}^{\min }$ é o menor escore observado para o j-ésimo fator e $F_{j}^{\max }$ é o maior escore observado para o jésimo fator.

O IDS agrega cinco importantes dimensões da saúde pública: recursos humanos e financeiros, atenção básica, atenção ambulatorial e hospitalar de média e alta complexidade, saúde materno infantil e vigilância em saúde ambiental. O índice varia entre 0 (valor mínimo) e 1 (valor máximo) e a classificação divide os municípios em três faixas: baixo desenvolvimento da saúde (IDS $\leq 0,33)$, médio $(0,33<$ IDS $\leq 0,67)$ e alto $(0,67<\operatorname{IDS} \leq 1)$.

\section{Análise de Conglomerados}

A técnica de análise de conglomerados é uma técnica estatística de interdependência que possibilita agrupar elementos em grupos homogêneos em função da similaridade dos valores das suas variáveis (FÁVERO et al., 2009). Após a escolha das variáveis, averiguação da necessidade ou não de padronizá-las e avaliação da manutenção ou exclusão de outliers, a próxima etapa da técnica refere-se à seleção da medida de proximidade a ser utilizada na pesquisa. Nesse estudo, foi utilizada a Distância Quadrática Euclidiana, adotada como padrão de distância no pacote estatístico SPSS. De acordo com Fávero et al. (2009), na Distância Quadrática Euclidiana, a distância entre duas observações ( $i$ e $j$ ) resulta da soma dos quadrados das diferenças entre $i$ e $j$ para todas as $p$ variáveis. É dada por: 


$$
d_{i j}^{2}=\sum_{k=1}^{p}\left(\begin{array}{ll}
x_{i k} & X_{j k}
\end{array}\right)^{2}
$$

Em que $x_{i k}$ é o valor da variável $k$ referente à observação $i$ e $x_{j k}$ representa a variável $k$ para a observação $j$. Conforme salienta Pfeiffer (1980), há dois grupos de métodos que podem ser utilizados para a execução do processo de agrupamento: o hierárquico e o não hierárquico. Nos métodos hierárquicos, os grupos são formados sobre níveis diferentes de distância ou semelhança, podendo ser divisivos ou aglomerativos. Os métodos não hierárquicos, por sua vez, caracterizam-se pelo agrupamento simultâneo dos elementos no número de grupos sugerido pelo pesquisador. Neste estudo foi utilizado um dos métodos de agrupamento hierárquico, o de maior distância ou ligação completa. Após o cálculo do IDS, foi utilizada a análise de conglomerados de modo a possibilitar o agrupamento dos municípios conforme o seu nível de desenvolvimento da saúde.

\section{Definição das variáveis}

Esta análise foi realizada com base em dados obtidos através do Sistema de Informação de Vigilância da Qualidade da Água para Consumo Humano (SISAGUA), Departamento de Informática do SUS (DATASUS) e Sistema de Informações sobre Orçamentos Públicos em Saúde (SIOPS) para o ano de 2011. Para a determinação dos fatores foram selecionadas, com base no estudo de Albuquerque et al. (2017), 15 variáveis que retratam as condições de saúde no semiárido brasileiro, listadas a seguir: percentual de realização das análises de vigilância da qualidade da água, quantidade de médicos por mil habitantes, quantidade de enfermeiros por mil habitantes; quantidade de outros profissionais de saúde por mil habitantes (fisioterapeuta, fonoaudiólogo, nutricionista, farmacêutico, assistente social, psicólogo, técnico de enfermagem e auxiliar de enfermagem), cobertura populacional estimada pelas Equipes Básicas de Saúde, cobertura populacional estimada pelas Equipes Básicas de Saúde Bucal, proporção de nascidos vivos de mães com 7 ou mais consultas de pré-natal, razão de exames citopatológicos do colo do útero em mulheres de 25 a 59 anos e a população da mesma faixa etária, razão de procedimentos ambulatoriais de média complexidade selecionados e população residente, razão de internações clínico-cirúrgicas de média complexidade e população residente, razão de procedimentos ambulatoriais de alta complexidade selecionados e população residente, razão de internações clínico-cirúrgicas de alta complexidade e população residente, proporção de internações sensíveis à atenção básica, despesa total com saúde por habitante e recursos transferidos do SUS por habitante.

\section{RESULTADOS E DISCUSSÃO}

\section{Análise Fatorial}

Com o objetivo de verificar se os dados suportam a Análise Fatorial foi feita a análise da matriz de correlações entre as variáveis, com o teste estatístico de esfericidade de Bartlett. Esse teste possibilita observar a probabilidade estatística da existência de correlações significativas entre pelo menos algumas variáveis. O valor obtido $(5154,795)$ para o teste foi significativo a $1 \%$. Isso significa que a hipótese nula de 
que a matriz de correlação é uma matriz identidade pode ser rejeitada, isto é, a hipótese de que as variáveis não são correlacionadas.

Para analisar a adequabilidade da análise fatorial, foi examinado o índice de Kaiser-Meyer-Olkim. O resultado obtido foi 0,741 , o que evidencia que a análise fatorial é apropriada. Também foi analisada a MSA, na matriz anti-imagem de correlações, para cada uma das variáveis em análise. Todas as variáveis apresentaram MSA superior a 0,5, o que indica a adequação dos dados à análise fatorial. A comunalidade, segundo Fávero et al. (2009), representa a proporção da variância de cada variável explicada pelo conjunto dos fatores selecionados. O seu valor mínimo aceitável é de 0,5. Neste estudo, todas as variáveis apresentam comunalidades com valor superior a 0,5. A Tabela 1, exposta a seguir, mostra os fatores extraídos e a variância explicada por cada um deles.

Tabela 1: Fatores extraídos pelo método dos componentes principais.

\begin{tabular}{|l|l|l|l|}
\hline \multirow{2}{*}{ Fator } & Autovalor & Variância Explicada & \multicolumn{1}{|l}{} \\
\cline { 3 - 4 } & & Inicial (\%) & Rotação (\%) \\
\hline 1 & 3,771 & 25,141 & 18,468 \\
\hline 2 & 2,060 & 13,736 & 14,781 \\
\hline 3 & 1,630 & 10,866 & 13,499 \\
\hline 4 & 1,316 & 8,771 & 10,854 \\
\hline 5 & 1,174 & 7,826 & 8,739 \\
\hline Total & & 66,341 & 66,341 \\
\hline
\end{tabular}

Através do método dos componentes principais foram extraídos cinco fatores com autovalores maiores que 1 , de modo que eles, em conjunto, explicam $66,34 \%$ da variância dos dados originais, conforme pode ser observado na Tabela 1, o que é um percentual significativo. Foi realizada a rotação ortogonal dos fatores através do método Varimax, com o objetivo de extremar os valores das cargas fatoriais, de forma que cada variável se associe a um fator, facilitando a sua interpretação. Após a rotação, os fatores, em conjunto, continuam a explicar $66,34 \%$ da variância total. A Tabela 2 mostra as cargas fatoriais das variáveis utilizadas nesse estudo. As variáveis que mais se relacionam com os fatores apresentam cargas fatoriais maiores que 0,5, isto é, cargas fatoriais mais elevadas indicam a existência de maior correlação entre os fatores e cada uma das 15 variáveis do estudo.

Com base na Tabela 2, pode-se observar que o Fator 1 relaciona-se fortemente com as variáveis quantidade de médicos por mil habitantes, quantidade de enfermeiros por mil habitantes, quantidade de outros profissionais de saúde por mil habitantes e recursos transferidos do SUS por habitante. De forma moderada, relaciona-se também com a variável despesa total com saúde por habitante. Esse fator contribui para explicar 18,468\% da variação comum. Verifica-se que o Fator 1 mantém relacionamento com as variáveis que captam a oferta de profissionais de saúde e de recursos para o setor sendo, portanto, denominado indicador de recursos humanos e financeiros.

De acordo com Oliveira (2013), as principais dificuldades do SUS estão relacionadas ao financiamento, recursos humanos e gestão. O subfinanciamento é considerado o aspecto mais grave do sistema. É importante ressaltar a necessidade de garantir uma maior eficiência na aplicação dos recursos existentes, o que resultaria na melhoria dos serviços prestados. 
Tabela 2: Cargas fatoriais das variáveis analisadas.

\begin{tabular}{|c|c|c|c|c|c|c|}
\hline \multirow[t]{2}{*}{ Variável } & \multirow{2}{*}{$\begin{array}{l}\text { Carga } \\
\text { Fatorial } \\
\text { Fator } 1\end{array}$} & \multirow{2}{*}{$\begin{array}{l}\text { Comunalidade } \\
\text { Fator } 2 \\
\end{array}$} & & & & \\
\hline & & & Fator 3 & Fator 4 & Fator 5 & \\
\hline Percentual de realização das análises de vigilância da qualidade da água & 0,116 & $-0,546$ & $-0,061$ & $-0,138$ & 0,587 & 680 \\
\hline Quantidade de médicos por mil habitantes & 0,814 & $-0,027$ & 0,178 & 0,095 & 0,024 & ,705 \\
\hline Quantidade de enfermeiros por mil habitantes & 0,787 & 0,074 & $-0,055$ & 0,005 & 0,094 & ,636 \\
\hline Quantidade de outros profissionais por mil habitantes & 0,748 & 0,113 & 0,278 & 0,106 & $-0,072$ & ,666 \\
\hline Cobertura populacional estimada pelas equipes básicas de saúde & 0,138 & 0,842 & $-0,052$ & $-0,07$ & 0,144 & ,755 \\
\hline Cobertura populacional estimada pelas equipes básicas de saúde bucal & 0,163 & 0,856 & 0,043 & $-0,053$ & 0,138 & ,783 \\
\hline Proporção de nascidos vivos de mães com mais de 7 consultas & 0,074 & 0,165 & 0,17 & $-0,139$ & 0,723 & ,603 \\
\hline $\begin{array}{l}\text { Razão de exames citopatológicos do colo do útero em mulheres de } 25 \\
\text { a } 59 \text { anos e a população da mesma faixa etária }\end{array}$ & 0,006 & 0,4 & $-0,036$ & 0,272 & 0,584 & ,576 \\
\hline $\begin{array}{l}\text { Razão de procedimentos ambulatoriais de média complexidade } \\
\text { selecionados e população residente }\end{array}$ & 0,25 & $-0,168$ & 0,653 & 0,165 & 0,009 & ,544 \\
\hline $\begin{array}{l}\text { Razão de internações clínico cirúrgicas de média complexidade e } \\
\text { população residente }\end{array}$ & 0,09 & $-0,088$ & 0,079 & 0,845 & 0,059 & ,740 \\
\hline $\begin{array}{l}\text { Razão de procedimentos ambulatoriais de alta complexidade } \\
\text { selecionados e população residente }\end{array}$ & 0,213 & 0,056 & 0,826 & $-0,002$ & $-0,002$ & ,730 \\
\hline $\begin{array}{l}\text { Razão de internações clínico cirúrgicas de alta complexidade e } \\
\text { população residente }\end{array}$ & 0,068 & 0,136 & 0,827 & $-0,117$ & 0,129 & ,738 \\
\hline Proporção de internações sensíveis à atenção básica & $-0,015$ & 0,011 & $-0,055$ & 0,846 & $-0,123$ & ,735 \\
\hline Despesa total com saúde por habitante & 0,504 & 0,444 & 0,189 & $-0,117$ & 0,03 & ,501 \\
\hline Recursos transferidos do SUS por habitante & 0,7 & 0,127 & 0,192 & $-0,047$ & 0,112 & ,558 \\
\hline
\end{tabular}

A carência de profissionais de saúde é vista pela população brasileira como o principal problema do SUS (IPEA, 2011). No entanto, além da questão da escassez da força de trabalho, há também os problemas relacionados à distribuição e à qualificação dos profissionais. Uma melhor gestão dos recursos humanos e financeiros do SUS é crucial para o alcance da excelência na prestação de serviços de saúde à população.

O Fator 2 explica $14,781 \%$ da variação comum e apresenta uma forte relação com as variáveis cobertura populacional estimada pelas Equipes Básicas de Saúde e cobertura populacional estimada pelas Equipes Básicas de Saúde Bucal. Desse modo, esse fator foi chamado de indicador de acesso potencial à Atenção Básica.

A Atenção Básica brasileira é estruturada, em grande parte, pela estratégia Saúde Mais Perto de Você, um grupo de iniciativas voltadas ao cuidado da população no local em que vive, no qual estão incluídos, por exemplo, a Estratégia Saúde da Família, a Política Nacional de Alimentação e Nutrição, o Brasil Sorridente e o Programa Saúde na Escola (BRASIL, 2012).

Apesar de a Atenção Básica ter avançado e proporcionado melhoras na saúde da população nos últimos anos, muitos problemas persistem. Pinto (2011) cita os principais desafios a serem enfrentados: subfinanciamento, infraestrutura inapropriada, acesso restrito, baixa qualidade dos serviços, reduzida atuação na promoção à saúde e na expansão de ações intersetoriais, dificuldade para progredir na mudança do modelo de atenção e na qualificação da administração, condições e relações de trabalho precárias, escassez de profissionais com formação específica para atuar na Atenção Básica e necessidade de expandir a legitimidade da Atenção Básica junto à sociedade.

O Fator 3 está fortemente relacionado às variáveis razão de procedimentos ambulatoriais de média complexidade selecionados e população residente, razão de procedimentos ambulatoriais de alta complexidade selecionados e população residente e razão de internações clínico-cirúrgicas de alta complexidade e população residente. Desse modo, foi denominado indicador de acesso obtido na atenção 
ambulatorial de média e alta complexidade e na atenção hospitalar de alta complexidade. Esse fator explica $13,499 \%$ da variação comum.

De acordo com Solla et al. (2012), os serviços de média complexidade no Brasil são caracterizados pela dificuldade de acesso e baixa capacidade resolutiva, sobreposição da oferta de serviços na esfera ambulatorial e hospitalar, centralização em locais com elevada densidade demográfica e reduzido grau de integração entre as ações dos demais níveis de atenção à saúde. É importante salientar que as restrições relacionadas à oferta de serviços ambulatoriais de média complexidade conduziram, de certo modo, à elevação da demanda de alta complexidade, repercutindo negativamente sobre a população e sobre os custos do sistema.

Observando a Tabela 2, pode-se observar que o Fator 4 apresenta uma forte associação com as variáveis razão de internações clínico cirúrgicas de média complexidade e população residente e proporção de internações sensíveis à atenção básica e, por isso, foi chamado de indicador de internações. Esse fator explica $10,854 \%$ da variação comum.

O indicador proporção de internações sensíveis à atenção básica, que tem como finalidade mensurar a quantidade de internações que poderiam ter sido evitadas através da execução de ações mais aprimoradas nesse nível de atenção à saúde, é uma das formas mais disseminadas de avaliar a efetividade do cuidado na AB (BRASIL, 2013a; IPEA, 2015).

Diversos trabalhos nacionais e internacionais mostram que elevadas taxas de internação por condições sensíveis à atenção básica estão associadas às dificuldades de acesso aos serviços básicos de saúde e à qualidade dos mesmos. Entre os estudos nacionais podem ser citados os de Elias et al. (2008) e Guanais et al. (2009). As pesquisas de Bermudez et al. (2005) e Ansari et al. (2006) destacam-se como referências internacionais sobre $o$ assunto.

O Fator 5 está fortemente associado à variável proporção de nascidos vivos de mães com 7 ou mais consultas de pré-natal. Apresenta associação moderada com as variáveis razão de exames citopatológicos do colo do útero em mulheres de 25 a 59 anos e a população da mesma faixa etária e percentual de realização das análises de vigilância da qualidade da água. Assim, esse fator foi denominado indicador de atenção integral à saúde da mulher e da criança e de redução dos riscos e agravos à saúde da população. O mesmo contribui para explicar $8,739 \%$ da variação comum.

A variável proporção de nascidos vivos de mães com 7 ou mais consultas de pré-natal é ideal para avaliar as condições de acesso das gestantes ao atendimento pré-natal e a qualidade do mesmo em associação com outras variáveis, como taxa de mortalidade materna e infantil (BRASIL, 2013a). Atualmente, é um dos indicadores selecionados para verificar o cumprimento da meta 3.7 do ODS, que consiste no compromisso dos governos integrantes das Nações Unidas de assegurar o acesso universal aos serviços de saúde sexual e reprodutiva até 2030 (PNUD, 2015).

O número de consultas de pré-natal está diretamente associado ao melhor desempenho dos indicadores de saúde materno infantil. Diversos estudos nacionais (Schoeps et al., 2007; Domingues et al., 
2012) e internacionais (BARROS et al., 2010; DEBIEC et al., 2010) mostram que as intervenções realizadas na assistência pré-natal são efetivas para prevenir a morbimortalidade materna, perinatal e neonatal.

Coimbra et al. (2003), ao realizarem um estudo com o objetivo de identificar fatores relacionados ao uso inadequado da assistência pré-natal, concluíram que o mesmo está associado a várias causas indicativas da permanência de desigualdades sociais, como menor grau de escolaridade e baixa renda familiar.

O câncer de colo de útero e o câncer de mama estão entre os principais motivos de mortes de mulheres de 30 a 69 anos de idade por neoplasias no Brasil. Diante da necessidade de modificar esse cenário, o SUS deu prioridade às ações de rastreamento para detecção precoce do câncer uterino e do câncer de mama através do direcionamento de recursos financeiros adicionais para a execução do exame citopatológico, também conhecido como exame de Papanicolau, e da mamografia bilateral (IPEA, 2015).

Outra medida que merece destaque é a campanha de vacinação gratuita contra o Papilomavirus Humano (HPV) nas escolas e nos postos de saúde, com o objetivo de diminuir a incidência do câncer de colo do útero, visto que o mesmo está fortemente relacionado à infecção causada por subtipos oncogênicos do HPV (IPEA, 2015). Garantir a disponibilidade e a gestão sustentável da água e saneamento para todos é o sexto ODS, que compreende, entre diversas metas, o acesso universal e equitativo à água potável e a melhora da qualidade da água (PNUD, 2015).

A água é um elemento fundamental para o desenvolvimento sustentável. Os recursos hídricos e os serviços associados aos mesmos contribuem para proporcionar a redução da pobreza, para o desenvolvimento econômico e para a sustentabilidade do meio ambiente (UNESCO, 2015). A ausência de fornecimento de água potável, de saneamento e de higiene acarreta uma repercussão extremamente negativa na saúde e no bem-estar da sociedade além de prejuízos consideráveis na atividade econômica (PNUD, 2015). A variável percentual de realização das análises de vigilância da qualidade da água é essencial para avaliar a proporção de análises realizadas em amostras de água para consumo humano, possibilitando que sejam efetuadas inferências sobre a qualidade da água consumida pela população (BRASIL, 2016a).

\section{Índice de desenvolvimento da saúde}

Em virtude da dificuldade para analisar, agrupar e hierarquizar os municípios do semiárido brasileiro com relação ao desenvolvimento da saúde empregando apenas os valores dos escores fatoriais foi utilizado o Índice de Desenvolvimento da Saúde (IDS), calculado a partir da agregação dos cinco fatores extraídos, com base na equação 5 .

As informações pertinentes à distribuição absoluta e relativa dos municípios do semiárido brasileiro segundo as faixas do IDS são apresentadas na Tabela 3. Pode-se observar que uma minoria ínfima dos municípios da região está enquadrada nas faixas de médio e alto desenvolvimento da saúde. A expressiva maioria dos municípios apresenta baixo desenvolvimento da saúde.

A Tabela 4 expõe os municípios que se enquadram nas faixas de médio ou alto desenvolvimento da saúde e, portanto, apresentam os melhores resultados para o IDS. Os municípios com melhor desempenho 
do índice no semiárido brasileiro são divididos entre os estados do Ceará, Paraíba, Rio Grande do Norte e Bahia.

Tabela 3: Frequência absoluta e relativa dos municípios do semiárido brasileiro segundo as faixas do IDS, para o ano de 2011.

\begin{tabular}{|l|l|l|}
\hline \multirow{2}{*}{ Faixas do IDS } & Municípios & \multicolumn{1}{|l}{} \\
\cline { 2 - 3 } & Frequência Absoluta & Frequência Relativa (\%) \\
\hline Baixo & 1124 & 99,2 \\
\hline Médio & 7 & 0,6 \\
\hline Alto & 2 & 0,2 \\
\hline Total & 1133 & 100,0 \\
\hline
\end{tabular}

Tabela 4: Municípios do semiárido brasileiro com alto e médio IDS, para o ano de 2011.

\begin{tabular}{|c|c|c|c|}
\hline Faixas do IDS & Posição & Município & IDS \\
\hline \multirow[t]{2}{*}{ Alto } & 1 & Guamaré (RN) & 0,797 \\
\hline & 2 & Barbalha (CE) & 0,792 \\
\hline \multirow[t]{7}{*}{ Médio } & 3 & Piancó (PB) & 0,604 \\
\hline & 4 & Parari (PB) & 0,569 \\
\hline & 5 & Viçosa (RN) & 0,554 \\
\hline & 6 & Irecê (BA) & 0,536 \\
\hline & 7 & Sobral (CE) & 0,514 \\
\hline & 8 & São José do Brejo do Cruz (PB) & 0,482 \\
\hline & 9 & Guaramiranga (CE) & 0,481 \\
\hline
\end{tabular}

Ainda de acordo com a Tabela 4, pode-se observar que apenas dois municípios apresentam alto desenvolvimento, enquanto os demais registram médio desenvolvimento. A primeira colocação no ranking pertence a Guamaré $(0,7974)$, município localizado no litoral norte do Rio Grande do Norte e com população de 12.404 habitantes, segundo o Censo Demográfico de 2010 (IBGE, 2017). O município, conforme mostra o IFDM (Índice Firjan de Desenvolvimento Municipal) na área de saúde para o período de 2011 a 2103 , foi classificado na categoria de alto desenvolvimento, o que corrobora os resultados desta pesquisa (FIRJAN, 2015).

O município de Barbalha, segundo colocado no ranking, fica localizado na região sul do estado do Ceará e possui uma população de 55.323 habitantes, conforme o Censo Demográfico de 2010 (IPECE, 2017$).$ A cidade se destaca pelo elevado investimento em saúde, o que a transformou num importante polo regional de saúde, respondendo pela cobertura de municípios da região sul do Ceará e de estados vizinhos. Os seus hospitais públicos fazem parte da rede de assistência médica de alta complexidade do SUS e do governo estadual e fornecem suporte ao Hospital Regional do Cariri (NUNES et al., 2016). É importante ressaltar que, no período de 2008 a 2013, Barbalha também apresentou alto desenvolvimento, conforme o IFDM Saúde (FIRJAN, 2015).

Conforme pode ser visto na Tabela 4, somente sete municípios da região semiárida possuem médio desenvolvimento da saúde. Apesar de apresentarem um resultado modesto, os municípios mostram um bom desempenho em alguns indicadores como, por exemplo, número de enfermeiros por mil habitantes e cobertura populacional estimada pelas equipes básicas de saúde.

A debilidade da saúde pública no semiárido brasileiro, constatada pelo baixo IDS da grande maioria dos seus municípios, evidencia uma realidade que se repete em todo o país, sobretudo em localidades mais 
desfavorecidas. Dentro desse contexto, serão discutidas a seguir as particularidades da saúde pública na região, com base nas variáveis utilizadas para o cálculo do IDS.

Um dos grandes problemas da saúde pública é a escassez de médicos e a má distribuição deles em todo o país, o que acarreta a desigualdade na oferta e no atendimento. No semiárido, a proporção é de um médico por mil habitantes, considerada muito inferior ao necessário para a região. Analisando os municípios enquadrados na faixa de baixo desenvolvimento humano, a proporção é a mesma para a região semiárida como um todo, porém chama a atenção o fato de mais de $50 \%$ dos municípios apresentarem a relação de menos de um médico por mil habitantes.

Além do déficit de médicos, o Brasil também enfrenta a carência de profissionais da área de enfermagem. No semiárido brasileiro, a proporção é de 0,67 enfermeiro para cada mil habitantes. Considerando os municípios que apresentam baixo desenvolvimento da saúde, apenas $9,79 \%$ deles possuem um ou mais de um enfermeiro por mil habitantes. É relevante ressaltar que a proporção de enfermeiros por mil habitantes na região é inferior à proporção de médicos, o que torna a situação ainda mais preocupante, dada a importância do profissional de enfermagem para o sistema de saúde.

De acordo com o relatório da pesquisa Demografia Médica no Brasil, publicado em 2013, a concentração de médicos nas capitais coexiste com a má distribuição de outros profissionais da área e com o número insuficiente de estabelecimentos de saúde. De modo geral, os municípios que possuem mais condições de atração e fixação de médicos e demais profissionais são aqueles dotados de melhor infraestrutura na área de saúde, melhores condições coletivas de trabalho, nível de renda mais elevado e maior qualidade de vida (CFM, 2013).

A escassez de recursos na área de saúde pública constitui forte obstáculo para o alcance da universalidade, equidade e integralidade da atenção à saúde, princípios finalísticos do SUS (BRASIL, 2013b). O financiamento da saúde pública tem sido insuficiente para garantir os recursos financeiros necessários para atender às demandas de saúde. O subfinanciamento do SUS está associado à injustiça tributária, que contribui para a manutenção e aprofundamento da desigualdade social, à má distribuição das atribuições e recursos entre os entres federados e ao raciocínio financista que rege os investimentos no país (BATALHA, 2012).

A maneira como o financiamento da saúde é distribuído entre as esferas de governo constitui uma grave distorção, visto que os municípios, especialmente os de pequeno porte, ficam extremamente sobrecarregados, tanto pelo maior volume de despesas com a saúde como pelo menor nível de arrecadação nacional, o que os torna bastante dependentes de transferências estaduais e federais (BATALHA, 2012).

Com relação à atenção básica de saúde, as falhas existentes podem ser verificadas em todo o país, porém são mais graves nos municípios menos favorecidos, como é o caso daqueles que fazem parte do semiárido brasileiro. Além de serem verificados diversos problemas, como falta de medicamentos e escassez de materiais básicos para atendimento, uma auditoria do Tribunal de Contas da União constatou a falta de preparo daqueles que trabalham com esse nível de atenção à saúde. Aproximadamente $70 \%$ dos municípios 
brasileiros fiscalizados apresentam deficiências nas ações de treinamento e formação dos gestores e profissionais da atenção básica (TCU, 2015).

A falta de qualificação da força de trabalho prejudica consideravelmente a qualidade dos serviços prestados à sociedade, causando impactos negativos na qualidade de vida dos indivíduos e contribuindo para que não sejam verificadas melhorias em importantes indicadores de saúde. As deficiências existentes na atenção básica concorrem para a baixa resolutividade dos serviços oferecidos, impactando de forma prejudicial no desempenho do indicador proporção de internações por condições sensíveis à atenção básica (ISAB). Um percentual elevado para o mesmo evidencia que o número de internações clínicas poderia ser menor caso fossem desenvolvidas, nesse nível de atenção, ações mais qualificadas de promoção, prevenção, tratamento e reabilitação (BRASIL, 2013a).

Um dos maiores obstáculos a ser superado para o reestabelecimento do SUS é o desenvolvimento de redes assistenciais que assegurem tanto os serviços de atenção básica resolutivos como a continuação do cuidado, através da oferta adequada de serviços de média e alta complexidade, garantindo o acesso ao sistema conforme as necessidades da população (BRASIL, 2004a).

O acesso aos serviços de média e alta complexidade também constitui um problema grave e persistente, tanto para os municípios de pequeno porte, muitas vezes localizados em áreas geográficas de difícil acesso, como para os municípios que compõem regiões metropolitanas, visto que sua oferta está associada a custos elevados, maior densidade tecnológica e ao envolvimento de mão de obra especializada (BRASIL, 2004b).

A maioria dos municípios do semiárido brasileiro é de pequeno porte, não dispondo de recursos financeiros para ofertar serviços de média e alta complexidade. Além disso, é importante ressaltar que as populações desses municípios não são suficientes para justificar, a partir da perspectiva da relação custobenefício, a disponibilidade de serviços, equipamentos e instrumentais médico-hospitalares de alta densidade tecnológica (PIMENTA, 2016).

Nos sistemas de saúde, normalmente, o cuidado da mulher é priorizado na área de saúde reprodutiva, com ênfase na atenção pré-natal, parto, puerpério e planejamento reprodutivo, e na área de prevenção dos cânceres de colo de útero e de mama. Porém, no Brasil, o atendimento integral às mulheres ainda se encontra em processo de estabelecimento (BRASIL, 2016b).

Apesar do aumento considerável da cobertura da assistência pré-natal no Brasil, tornando-a praticamente universal, e da redução das desigualdades regionais e socioeconômicas no acesso às intervenções de saúde materna, a atenção pré-natal não apresenta padrão de qualidade satisfatório. A baixa qualidade da assistência pré-natal é observada em todas as regiões brasileiras, principalmente nas regiões Norte e Nordeste, sobretudo nas áreas rurais, ribeirinhas e de floresta, e em grupos populacionais que apresentam piores condições socioeconômicas (BRASIL, 2013c; VIELLAS et al., 2014; NUNES et al., 2016).

O câncer de colo do útero, também denominado cervical, é uma doença que poder ser amplamente prevenida, porém é uma das causas mais relevantes de morte de mulheres por câncer no mundo. Um programa integral de prevenção e controle do câncer de colo do útero, que objetive prevenir essa neoplasia 
maligna e reduzir suas taxas de morbidade e mortalidade, deve apresentar três componentes interdependentes: prevenção primária (redução do risco de infecção por HPV), prevenção secundária (rastreamento e tratamento das lesões precursoras) e prevenção terciária (tratamento oportuno e cuidados paliativos no câncer invasivo) (OPAS, 2016).

Maiores taxas de incidência e mortalidade do câncer cervical, verificadas predominantemente em populações que possuem piores condições socioeconômicas, estão fortemente relacionadas a disparidades existentes na prevenção, acompanhamento e tratamento (DOWNS et al., 2008). O acesso à água de qualidade e em quantidade suficiente é uma necessidade básica de todas as pessoas e um direito humano fundamental, que pode ser garantido à população através do fornecimento de serviços de abastecimento de água e saneamento confiáveis e acessíveis (PNUD, 2006; UNESCO, 2015).

A privação do acesso à água potável é um grave problema que persiste no Brasil, especialmente na zona rural de diversos municípios brasileiros, sobretudo naqueles que pertencem ao semiárido. Com o objetivo de minimizar os efeitos da seca no semiárido nordestino e norte de Minas Gerais, o governo federal, por meio da Operação Carro-Pipa, realiza a distribuição de água para a população em cisternas coletivas ou individuais através de caminhões-pipa (PORTAL BRASIL, 2016). As cisternas são entregues às famílias rurais por intermédio do Programa Cisternas, que têm como objetivo proporcionar o acesso à água para o consumo humano e para a produção de alimentos por meio da implementação de tecnologias sociais simples e de baixo custo (BRASIL, 2017).

Apesar da importância do programa de distribuição de cisternas para proporcionar mais qualidade de vida e saúde para a população, a iniciativa não é suficiente para solucionar a realidade vivenciada pelas famílias rurais. Além das dificuldades relacionadas à má qualidade da água utilizada, tanto fornecida pelos caminhões-pipa como captada da chuva, há o problema da falta de acesso a saneamento, que produz consequências graves na saúde, educação e economia.

Ao longo das três últimas décadas, tem havido um expressivo reconhecimento de que grande parte da carga global de doenças e as principais causas das iniquidades em saúde decorrem das condições sociais dos indivíduos. Tais condições são denominadas 'determinantes sociais da saúde' e compreendem os determinantes sociais, econômicos, políticos, culturais e ambientais da saúde (CSDH, 2008). Nessa perspectiva, a Tabela 5 expõe as médias dos indicadores socioeconômicos para cada uma das faixas do IDS.

Tabela 5: Médias dos indicadores socioeconômicos para o ano de 2010, por faixas do IDS.

\begin{tabular}{|c|c|c|c|}
\hline \multirow[t]{2}{*}{ Indicadores } & \multirow{2}{*}{$\begin{array}{l}\text { IDS } \\
\text { Baixo }\end{array}$} & \multirow[b]{2}{*}{ Médio } & \multirow[b]{2}{*}{ Alto } \\
\hline & & & \\
\hline Renda per capita & 273.42 & 330.98 & 385.20 \\
\hline$\%$ de extremamente pobres & 22.12 & 15.47 & 9.36 \\
\hline$\%$ de pobres & 40.71 & 33.93 & 26.07 \\
\hline Índice de Theil - L & 0.52 & 0.50 & 0.49 \\
\hline \% de empregados com carteira & 15.90 & 22.21 & 37.78 \\
\hline \% da população em domicílios com água encanada & 70.62 & 72.71 & 84.94 \\
\hline Taxa de analfabetismo - 15 anos ou mais & 27.76 & 21.24 & 20.79 \\
\hline IDHM & 0.59 & 0.63 & 0.65 \\
\hline IDHM Renda & 0.56 & 0.59 & 0.62 \\
\hline IDHM Longevidade & 0.76 & 0.76 & 0.79 \\
\hline IDHM Educação & 0.49 & 0.56 & 0.57 \\
\hline
\end{tabular}

Fonte: PNUD (2013b). 
Os resultados sugerem que nos municípios em que se verifica um maior nível de desenvolvimento da saúde, os indicadores socioeconômicos apresentam melhores resultados. Realizando uma comparação entre os três grupos, pode-se observar que os munícipios classificados com IDS alto apresentam, em termos médios, a maior renda per capita, a menor proporção de pobres e de extremamente pobres, o menor Índice de Theil-L, o maior percentual de empregos formais, a maior proporção da população em domicílios com água encanada, a menor taxa de analfabetismo e maior IDHM.

\section{CONCLUSÕES}

Esta pesquisa objetivou analisar o desempenho da saúde pública no semiárido brasileiro através do Índice de Desenvolvimento da Saúde, calculado a partir de um conjunto de variáveis relacionadas às condições de saúde da população.

Conforme esperado, a análise permitiu constatar que a grande maioria dos municípios apresenta um baixo desenvolvimento da saúde. Esse resultado, aliado às condições socioeconômicas desfavoráveis da região, sugere que a realização de investimentos para aumentar a oferta dos serviços de saúde e melhorar a qualidade dos mesmos é fundamental, mas não é suficiente para assegurar o desenvolvimento sustentável e uma melhor qualidade de vida e bem- estar para a população.

Considerando que os grupos populacionais que se encontram em situação de desvantagem socioeconômica apresentam piores condições de saúde quando comparados àqueles que estão em posições superiores, seja com relação à renda, educação, emprego ou habitação, é importante salientar que a efetividade das políticas públicas de saúde exige ações coordenadas nos diversos setores de ação governamental, especialmente naqueles cujo desempenho gera impactos significativos sobre a saúde.

\section{REFERÊNCIAS}

ALBUQUERQUE, C.; MARTINS, M.. Indicadores de desempenho no Sistema Único de Saúde: uma avaliação dos avanços e lacunas. Saúde debate, Rio de Janeiro, v.41, n.spe, p.118-137, 2017. DOI: http://dx.doi.org/10.1590/010311042017 s 10

ANSARI, Z.; LADITKA, J. N.; LADITKA, S. B.. Access to health care and hospitalization for ambulatory care sensitive conditions. Med Care Res Ver, v.63, n.6, p.719-41, 2006. DOI: https://doi.org/10.1177/1077558706293637

BARROS, F. C.; BHUTTA, Z. A.; BATRA, M.; HANSEN, T. N.; VICTORA, C. G.; RUBENS, C. E.. Global report on preterm birth and stillbirth (3 of 7): evidence for effectiveness of interventions. BMC Pregnancy and Childbirth, v.10, n.1, 2010. DOI: https://doi.org/10.1186/1471-2393-10-S1-S3

BATALHA, E.. Para entender o sub(financiamento) do SUS. Radis Comunicação e Saúde, Rio de Janeiro, n.116, 2012.

BERMUDEZ, D.; BAKER, L.. The relationship between SCHIP enrollment and hospitalizations for ambulatory care sensitive conditions in California. J Health Care Poor Underserved, v.16, n.1, p.96-110, 2005. DOI: https://doi.org/10.1353/hpu.2005.0003
BRASIL. Ministério da Saúde. Secretaria de Atenção à Saúde. Avaliação normativa do Programa Saúde da família no Brasil: monitoramento da implantação e funcionamento das equipes da saúde da família: 2001-2002. Brasília: Ministério da Saúde, 2004a.

BRASIL. Ministério da Saúde. Atenção Especializada em Saúde no Brasil: indicadores e tabelas selecionados. Brasília: Ministério da Saúde, 2004b.

BRASIL. Ministério da Saúde. Secretaria de Atenção à Saúde. PNAB: Política Nacional de Atenção Básica. Brasília: Ministério da Saúde, 2012.

BRASIL. Ministério da Saúde. Secretaria Executiva. Índice de Desempenho do Sistema Único de Saúde (IDSUS): Fichas Técnicas dos Indicadores. Brasília: Ministério da Saúde, 2013a.

BRASIL. Ministério da Saúde. Financiamento do SUS. Brasília: Ministério da Saúde, 2013b.

BRASIL. Ministério da Saúde. Secretaria de atenção à saúde. Atenção ao pré-natal de baixo risco. Brasília: MS; 2013c. 
BRASIL. Ministério da Saúde. Caderno de Diretrizes, Objetivos, Metas e Indicadores 2016. Brasília: Ministério da Saúde, 2016a.

BRASIL. Ministério da Saúde. Protocolos da Atenção Básica: Saúde das Mulheres. Ministério da Saúde, Instituto Sírio Libanês de Ensino e Pesquisa. Brasília: Ministério da Saúde, 2016b.

BRASIL. Ministério do Desenvolvimento Social. Programa Cisternas. Brasília: Ministério do Desenvolvimento Social, 2017.

BUSS, P. M.; CHAMAS, C.; FAID, M; MOREL, C.. Desenvolvimento, saúde e política internacional: a dimensão da pesquisa e inovação. Cad. Saúde Pública, Rio de Janeiro, v.32, n.2, 2016. DOI: http://dx.doi.org/10.1590/0103$\underline{311 \times 00046815}$

CFM. Conselho Federal de Medicina. Demografia Médica no Brasil. 2 ed. São Paulo: Conselho Regional de Medicina do Estado de São Paulo, 2013.

COIMBRA, L. C.; SILVA, A. A. M.; MOCHEL, E. G.; ALVES, M. T. S. S. B.; RIBEIRO, V. S.; ARAGÂO, V. M. F.; BETTIOL, H.. Fatores associados à inadequação do uso da assistência prénatal. Rev. Saúde Pública, São Paulo, v.37, n.4, p.456-462, 2003. DOI: http://dx.doi.org/10.1590/S0034$\underline{89102003000400010}$

CSDH. Commission on Social Determinants of Health. Closing the gap in a generation: health equity through action on the social determinants of health. Commission on Social Determinants of Health final report. Geneva: WHO, 2008.

DEBIEC, K. E.; PAUL, K. J.; MITCHELL, C. M.; HITTI, J. E.. Inadequate prenatal care and risk of preterm delivery among adolescents: a retrospective study over 10 years. Am J Obstet Gynecol., v.203, n.2, p.122, 2010. DOI: https://doi.org/10.1016/j.ajog.2010.03.001

DOMINGUES, R. M. S. M.; HARTZ, Z. M. A.; DIAS, M. A. B.; LEAL, M. C.. Avaliação da adequação da assistência pré-natal na rede SUS do Município do Rio de Janeiro, Brasil. Cad. Saúde Pública, Rio de Janeiro, v.28, n.3, p.425-437, 2012. DOI: http://dx.doi.org/10.1590/S0102-311X2012000300003

DOWNS, L.; SMITH, J. S.; SCARINCI, I.; FLOWERS, L.; PARHAM, G.. The disparity of cervical cancer in diverse populations. Gynecologic Oncology, v.109, n.2, p.s22-s30, 2008. DOI: https://doi.org/10.1016/j.ygyno.2008.01.003

ELIAS, E.; MAGAJEWSKI, F.. A Atenção Primária à Saúde no sul de Santa Catarina: uma análise das internações por condições sensíveis à atenção ambulatorial, no período de 1999 a 2004. Rev. bras. epidemiol., São Paulo, v.11, n.4, p.633-647, 2008. DOI: http://dx.doi.org/10.1590/S1415790X2008000400011

FÁVERO, L. P.; BELFIORE, P.; SILVA, F. L.; CHAN, B. L.. Análise de dados: modelagem multivariada para tomada de decisões. Rio de Janeiro: Elsevier, 2009.
FIRJAN. Federação das Indústrias do Estado do Rio de Janeiro. Índice Firjan de Desenvolvimento Municipal (IFDM). 2015.

GUANAIS, F.; MACINKO, J.. Primary care and avoidable hospitalizations: evidence from Brazil. J Ambul Care Manage, v.32, n.2, p.115-22, 2009. DOI: https://doi.org/10.1097/JAC.0b013e31819942e51

HAIR, J. F.; BLACK, W. C.; BABIN, B. J.; ANDERSON, R. E.; TATHAM, R. L.. Análise multivariada de dados. 5 ed. Porto Alegre: Bookman, 2005.

IBGE. Instituto Brasileiro de Geografia e Estatística. Guamaré. Rio de Janeiro: IBGE, 2017.

IPEA. Instituto de Pesquisa Econômica Aplicada. Sistema de indicadores de percepção social (SIPS): Saúde. Brasília: IPEA, 2011.

IPEA. Instituto de Pesquisa Econômica Aplicada. Políticas sociais: acompanhamento e análise. BPS n.23. Brasília: IPEA, 2015.

IPECE. Instituto de Pesquisa e Estratégia Econômica do Ceará. Perfil Básico Municipal 2016: Barbalha. Fortaleza, IPECE: 2017.

JOHNSON, R. A.; WICHERN, D. W.. Applied multivariate statistical analysis. 6 ed. Upper Saddle River: Pearson Education, 2007.

LEMOS, J. J. S.. Indicadores de Degradação no Nordeste Subúmido e Semi-árido. Revista SOBER, 2000.

MENDONÇA, C. S.; HARZHEIM, E.; DUNCAN, B. B.; NUNES, L. $\mathrm{N}$.; LEYH, W.. Trends in hospitalizations for primary care sensitive conditions following the implementation of Family Health Teams in Belo Horizonte, Brazil. Health Policy Plan., v.27, n.4, p.348-55, 2012. DOI:

https://doi.org/10.1093/heapol/czr043

NUNES, J. T.; GOMES, K. R. O.; RODRIGUES, M. T. P.; MASCARENHAS, M. D. M.. Qualidade da assistência pré-natal no Brasil: revisão de artigos publicados de 2005 a 2015. Cad. Saúde Colet., Rio de Janeiro, v.24, n.2, p.252-261, 2016. DOI: http://dx.doi.org/10.1590/1414-462X201600020171

NUNES, E. S.; SOUSA, E. P.. Análise da eficiência no gerenciamento público com a saúde para os municípios cearenses. In: Encontro Economia do Ceará em Debate, 12. Anais. Fortaleza: IPECE, 2016.

OPAS. Organização Pan-Americana da Saúde. Controle integral do câncer do colo do útero. Guia de práticas essenciais. Washington: OPAS, 2016.

PFEIFFER, D.. Disparidades de desenvolvimento no Brasil: um exemplo da análise de cluster. Revista Brasileira de Estatística, Rio de janeiro, v.41, n.164, p.559-576, 1980.

PIMENTEL, V. P.; GOMES, R. P.; LANDIM, A. B.; PIERONI, J. P.; PALMEIRA FILHO, P. L. Saúde como desenvolvimento: perspectivas para atuação do BNDES complexo industrial da saúde. In: BNDES 60 anos: perspectivas setoriais. Rio de Janeiro: Banco Nacional de Desenvolvimento Econômico e Social, 2012. p.300-332. 
PINTO, H.. Nova Política Nacional de Atenção Básica: mais acesso e qualidade. Brasília: Ministério da Saúde, 2011.

PNUD. Programa das Nações Unidas para o Desenvolvimento. Relatório do Desenvolvimento Humano 2006. Brasília: PNUD, 2006.

PNUD. Programa das Nações Unidas para o Desenvolvimento. Relatório do Desenvolvimento Humano 2013. Brasília: PNUD, 2013a.

PNUD. Programa das Nações Unidas para o Desenvolvimento. Atlas do Desenvolvimento Humano no Brasil 2013. Brasília: PNUD, 2013b.

PNUD. Programa das Nações Unidas para o Desenvolvimento. Acompanhando a agenda $\mathbf{2 0 3 0}$ para o desenvolvimento sustentável: subsídios iniciais do Sistema das Nações Unidas no Brasil sobre a identificação de indicadores nacionais referentes aos objetivos de desenvolvimento sustentável. Programa das Nações Unidas para o Desenvolvimento. Brasília: PNUD, 2015.

SÁ, V. C.; SOUZA, B. I.. Convivência com o semiárido: Desafios e possibilidades de uma comunidade rural. Revista Globalização, Competitividade e Governabilidade, v.6, n.2, p.46-65, 2012. DOI: http://dx.doi.org/10.3232/GCG.2012.V6.N2.03
SACHS, J.. Macroeconomics and Health: investing in health for economic development. Genebra: FMI, 2001.

SCHOEPS, D.; ALMEIDA, M. F.; ALENCAR, G. P.; FRANÇA JR., I.; NOVAES, H. M. D.; SIQUEIRA, A. A. F.; CAMPBELL, O.; RODRIGUES, L. C.. Fatores de risco para mortalidade neonatal precoce. Rev. Saúde Pública, São Paulo, v.41, n.6, p.1013-1022, 2007. DOI: http://dx.doi.org/10.1590/S0034$\underline{89102007000600017}$

SOLLA, J.; CHIORO, A.. Atenção ambulatorial especializada. In: GIOVANELLA, L.; ESCOREL, S.; LOBATO, L. V. C.; NORONHA, J. C.; CARVALHO, A. I.. Políticas e sistemas de saúde no Brasil. 2 ed. Rio de Janeiro: Fiocruz, 2012. cap.17, p.547-576.

TCU. Tribunal de Contas da União. Gestão da atenção básica à saúde no Brasil: auditoria coordenada. Brasília: TCU, Secretaria de Controle Externo da Saúde, 2015.

UNESCO. Organização das Nações Unidas para a Educação, a Ciência e a Cultura. Relatório Mundial das Nações Unidas sobre o Desenvolvimento dos Recursos Hídricos: Água para um Mundo Sustentável. Colombella: Perugia, 2015.

WHO. World Health Organization. The World Health Report 2010. Health Systems Financing: The Path to Universal Coverage. Geneva: WHO, 2010.

A CBPC - Companhia Brasileira de Produção Científica (CNPJ: 11.221.422/0001-03) detém os direitos materiais desta publicação. Os direitos referem-se à publicação do trabalho em qualquer parte do mundo, incluindo os direitos às renovações, expansões e disseminações da contribuição, bem como outros direitos subsidiários. Todos os trabalhos publicados eletronicamente poderão posteriormente ser publicados em coletâneas impressas sob coordenação da Sustenere Publishing, da Companhia Brasileira de Produção Científica e seus parceiros autorizados. Os (as) autores (as) preservam os direitos autorais, mas não têm permissão para a publicação da contribuição em outro meio, impresso ou digital, em português ou em tradução. 